\title{
Cecal Volvulus: A Case Report and Review of the Literature
}

\author{
Binyam Yohannes \\ Mahteme Bekele Muleta if \\ Department of Surgery, St. Paul's \\ Hospital Millennium Medical College, \\ Addis Ababa, Ethiopia
}

Background: Cecal volvulus is a rare cause of adult intestinal obstruction occurring 2.87.1 persons per million per year. Its rarity and the variety of clinical symptoms may lead to a delay in the diagnosis and appropriate intervention.

Case Presentation: Here, we present a 25-year old female patient from a rural area who presented with a 3-day history of colicky abdominal pain and vomiting of bilious intestinal content; she had also developed abdominal distension, failed to pass feces, and flatus. She underwent emergency laparotomy, where the intraoperative finding was a perforated 360 degrees counterclockwise volvulated cecum, and a right hemicolectomy was performed with primary ileotransverse anastomosis.

Discussion: Cecal volvulus is the axial rotation of the cecum, ascending colon, and terminal ileum. This can result either in complete or partial obstruction of the affected segment of the intestine with impending vascular compromise and gangrene, which mandates a timely diagnosis and intervention.

Conclusion: Cecal volvulus is a rare cause of adult intestinal obstruction, and urgent surgical intervention saves the life of patients.

Keywords: cecal volvulus, intestinal obstruction, colicky abdominal pain, hemi-colectomy

\section{Introduction}

Colonic Volvulus is defined as a twisted loop of the bowel and its mesentery on a fixed point at the base and it may occur in the sigmoid colon, cecum, splenic flexure and the descending colon. ${ }^{1}$ Cecal volvulus is an uncommon cause of intestinal obstruction, and is the axial twisting of the cecum, ascending colon, terminal ileum, and their associated mesenteries as pedicles. ${ }^{1}$ It is estimated that it occurs in 2.8-7.1 per million people per year. ${ }^{2}$ It is an infrequent cause of intestinal obstruction, accounting for $1-5 \%$ of all adult intestinal obstructions; and up to $40 \%$ of colonic volvulus. ${ }^{3}$

A mobile/non-fixed cecum is mentioned as one significant associated finding in patients with cecal volvulus, and this was seen in about $25 \%$ of cadaveric studies. ${ }^{4}$ Its variable clinical symptoms make it difficult to diagnose early.

Here we reported a case of acute abdomen in a 25 year-old female patient who was operated on for the diagnosis of acute abdomen secondary to intestinal obstruction with an intraoperative finding of a perforated cecal volvulus.

\section{Case Report}

A 25 year-old housewife visited our emergency surgical department with a 3-day history of colicky abdominal pain associated with frequent bilious vomiting. She also
Correspondence: Binyam Yohannes

Tel +2519l 1463939

Email binyam@gmail.com (c) (7) ( 2021 Yohannes and Muleta. This work is published and licensed by Dove Medical Press Limited. The full terms of this license are available at https://www.dovepress. (c) ${ }_{\mathrm{BY}} \mathrm{NC}$ com/terms.php and incorporate the Creative Commons Attribution - Non Commercial (unported, v3.0) License (http://creativecommons.org/licenses/by-nc/3.0/). By accessing the work you hereby accept the Terms. Non-commercial uses of the work are permitted without any further permission from Dove Medical Press Limited, provided the work is properly attributed. For permission for commercial use of this work, please see paragraphs 4.2 and 5 of our Terms (https://www.dovepress.com/terms.php). 
reported a progressive abdominal distension of 2 days prior to her presentation, along with failure to pass feces and flatus. Otherwise she did not have a previous history of similar complaints or constipation, no weight loss or bleeding from the rectum, no groin swelling, no prior abdominal surgery, or endoscopic procedures. She is a mother of three children, the youngest being 6 months old.

Upon examination she was acutely sick looking, dehydrated, with a dry tongue and buccal mucosa. Her vital signs were deranged, with a pulse rate of 110 beats per minute, blood pressure $110 / 60 \mathrm{mmHg}$, but afebrile $\mathrm{T}^{0}=36.9^{\circ} \mathrm{C}$ and oxygen saturation of $94 \%$ with atmospheric oxygen. She had a grossly distended abdomen with visible intestinal loops in the central part of her abdomen, hyperactive bowel sound, and mild tenderness over the mid-abdomen. Digital rectal examination revealed an empty ballooned rectum.

Her baseline investigation showed a white blood cell count of $13,000 / \mu \mathrm{L}$ with polymorph neutrophils accounting for $69 \%$, and her blood group was shown to be $\mathrm{O}$ positive. A plain abdominal X-ray (Figure 1) showed a markedly distended large bowel loop with long axis oriented from the right lower quadrant to the midabdomen with a single air fluid level characteristic of cecal volvulus.

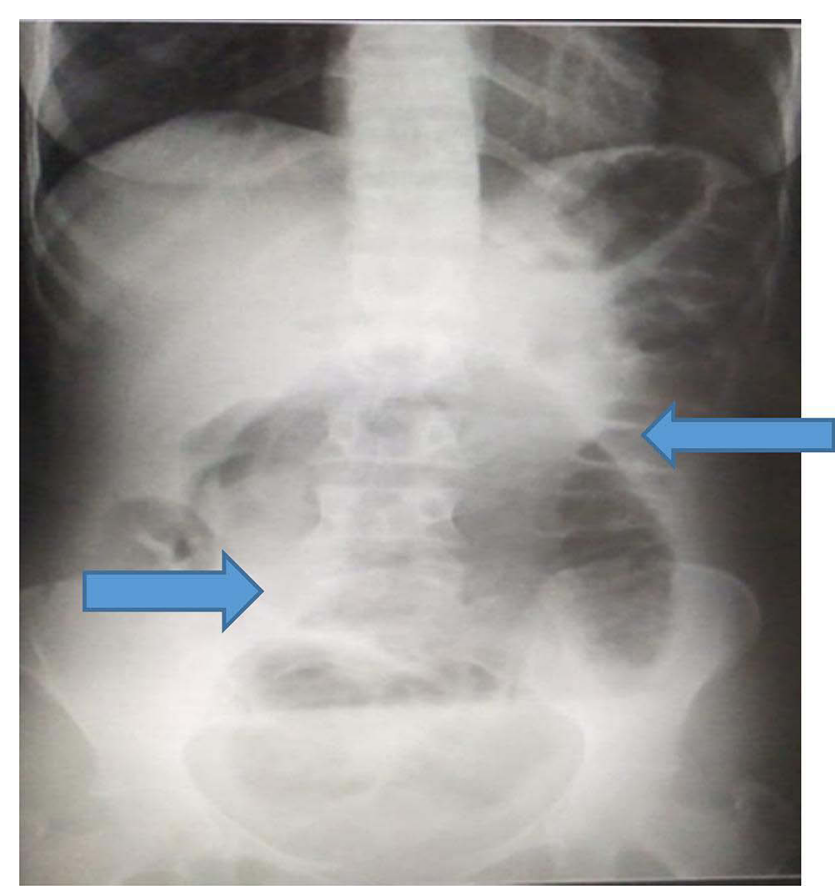

Figure I A dilated large bowel loops in the central abdomen its apex pointing to the left upper quadrant (right arrow) and peripherally displaced small bowel loops (left arrow).

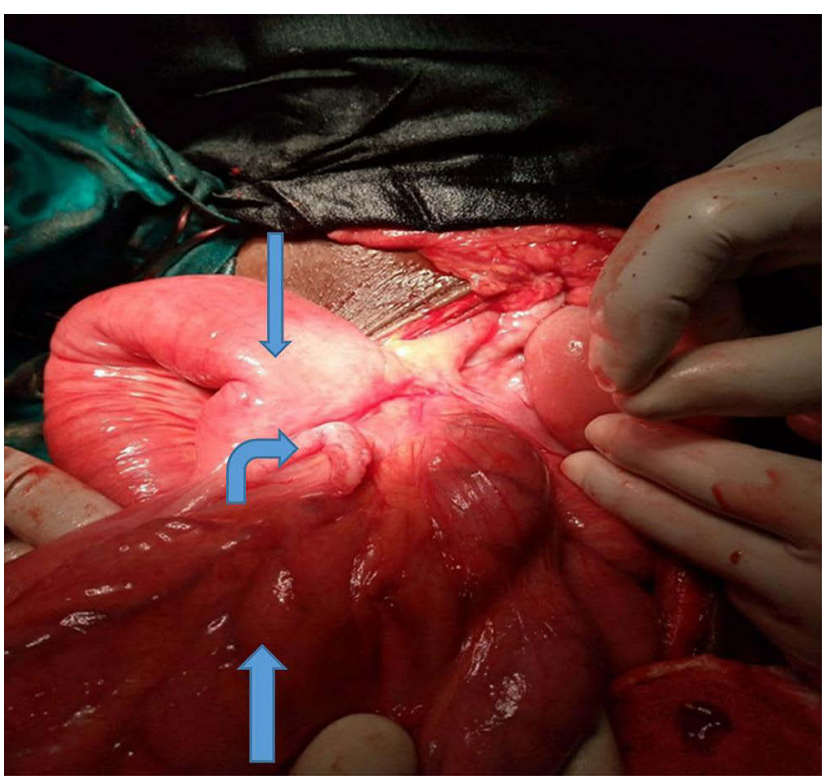

Figure 2 Intraoperative finding showing a distended, mobile and volvulated cecum (up arrow), distal ileum (down arrow) and the appendix (bent arrow).

She was admitted, resuscitated with intravenous fluid administration, preoperative preparations were made, informed consent was obtained for the procedure, she was then transferred to the operating theater, under general anesthesia and endotracheal intubation, the abdomen was cleaned with Povidone iodine and, through a vertical midline incision, a laparotomy was performed. The intraoperative findings were hugely distended bowel loops, 360 degrees anticlockwise volvulated mobile cecum with a contained perforation on the anti-mesenteric border with proximally distended small bowel loops and distally collapsed colon (Figures 2 and 3).

Otherwise, there was no other intra-abdominal pathology discovered. We did a right hemi-colectomy with bowel continuity maintained by end to side ileotransverse anastomosis.

The patient had an uneventful postoperative course, she was started on a fluid diet on the 3rd post-operative day and advanced to a solid diet on the 5th day and was discharged with improvement on the 7th postoperative day. She had a follow-up at 6 months in the surgical referral clinic with no complications.

\section{Discussion}

Cecal volvulus is the axial rotation of the ascending colon, cecum, and terminal ileum. It wass first described by Rokitansky in $1837 .^{5}$ Cecal volvulus (CV) could be divided into two major groups of loop axial ileocolic, the 


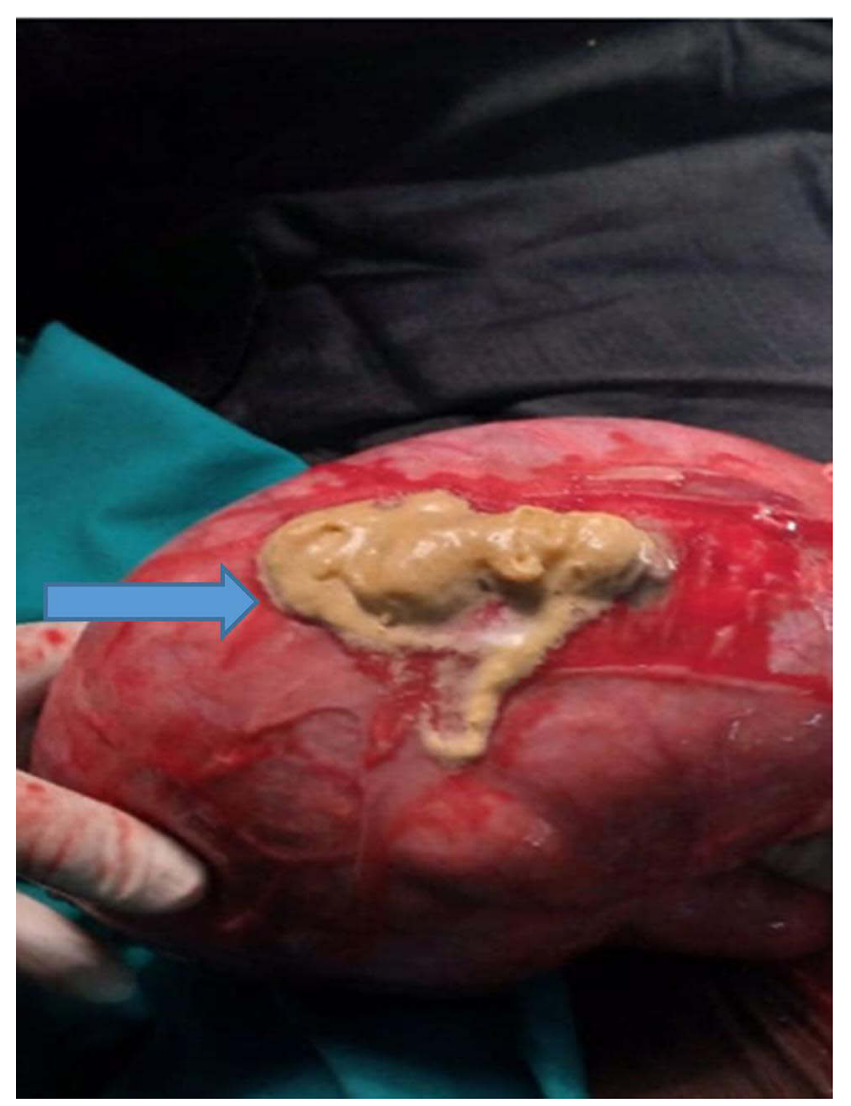

Figure 3 Intraoperative finding depicting a thinned out and perforated cecum with fecal matter oozing (right arrow).

commonest, accounting for $90 \%$ of cases, which can appear as a clockwise or counter clockwise pattern in which the latter one is the most commonly seen variety, our patient also presented with the counterclockwise pattern. ${ }^{4}$ Cecal bascule is where there is an upward folding of the cecum either anteriorly or posteriorly. ${ }^{1}$

Cecal volvulus is said to be associated with inadequate right colon fixation or anomalies in which the right colon does not properly fuse to the lateral peritoneum. ${ }^{6}$ Other well documented predisposing factors for $\mathrm{CV}$ are long-standing constipation, distal obstruction, previous abdominal surgeries, intraabdominal masses, being bedridden, and undergoing colonoscopy procedures. ${ }^{1,7}$ When it occurs in the younger populations its more commonly caused by post-OP adhesions, pregnancy, or aganganglionic megacolon ${ }^{7-9}$ Reports have shown that there is no sex predilection and the mean age of incidence is 61.8 years. $^{6}$ Our patient is young and did not have any of the abovementioned predisposing factors.

The clinical presentation depends on the duration of the complaints and the presence of complications. Symptoms of abdominal pain, associated with vomiting and abdominal distension, are reported as the commonest presentations; ${ }^{1}$ all the mentioned symptoms were documented in our patient. Preoperative diagnosis of $\mathrm{CV}$ poses a significant challenge because of its rarity and nonspecific symptoms, mostly diagnosed intraoperatively. ${ }^{10}$ We were also able to reach the diagnosis intraoperatively.

Laboratory investigations are neither specific nor sensitive for the diagnosis of $\mathrm{CV}$ but may suggest the degree of obstruction and presence of complications. ${ }^{11}$ Our patient had an elevated leukocytosis level with predominant neutrophil count which can be explained by the presence of a perforated cecum.

Radiologic imaging may be abnormal and detect $\mathrm{CV}$ in $45-56 \%$ of the cases. ${ }^{1}$ Plain abdominal $x$-ray is highly sensitive for the diagnosis of $\mathrm{CV}$ with the characteristic "coffee bean" sign deformity, its apex pointing to the left upper quadrant; ${ }^{9}$ which was also seen in our patient. Other findings commonly seen are cecal dilatation (98-100\%), single air-fluid level (72-88\%), small bowel dilatation (42-55\%), and absence of gas in the distal colon (82\%). $\mathrm{CT}$ is more sensitive and specific for diagnosing $\mathrm{CV}$ and detecting complications. ${ }^{10}$

Once the diagnosis of CV was made patients should undergo urgent laparotomy or laparoscopic procedures to untwist the segment early to avoid the risk of strangulation. ${ }^{1}$ Once exploration has been done and if the intraoperative finding is a volvulated cecum; its viability should be assessed. Resection (right hemicolectomy) for all gangrenous and perforated or grossly distended thinned out cecal volvulus is the treatment of choice with primary anastomosis, ileostomy with mucous fistula is depending on the patient's intraoperative physiology and bowel condition, resection has the absolute advantage of eliminating the risk of recurrence. ${ }^{1}$ Our patient had a perforated cecum with minimal contamination and she underwent a right hemicolectomy with ileo-transverse primary anastomosis.

Manual detorsion without resection options is not advisable since it is associated with an unacceptable risk of recurrence $(40 \%){ }^{5}$ Cecopexy or cecostomy are also available options depending on the overall status of the patient. Possible endoscopic detorsion in cecal volvulus is an emerging option for non-strangulated volvulus but associated with high risk of recurrence. ${ }^{12}$

\section{Conclusion}

Cecal volvulus is a rare cause of adult intestinal obstruction with non-specific symptoms and laboratory findings. Its rarity makes its preoperative diagnosis challenging and hampers early intervention, resulting in a high incidence of 
complications and mortality, hence a high index of suspicion and urgent intervention is needed. Surgery is the mainstay of management for $\mathrm{CV}$.

Resection with hemicolectomy is the accepted method of treatment for gangrenous or perforated cecal volvulus.

High quality studies including case series studies might be more helpful in the understanding of $\mathrm{CV}$.

\section{Ethical Approval and Consent}

Written informed consent was obtained from the patient for publication of this case report and accompanying images considering its publication would help to understand the case of appendiceal agenesis well. A copy of the written consent is available for review from the Editor-inChief of this journal on request. Ethical approval from the Institutional review board is not required given it is a case report.

\section{Acknowledgment}

We want to thank our patient and her family for consenting to the publication of the article.

\section{Author Contributions}

All authors made a significant contribution in the conception, execution, took part in drafting the case report, critically reviewing the article; gave final approval of the version to be published; have agreed on the journal to which the article has been submitted; and agree to be accountable for all aspects of the work.

\section{Funding}

There is no funding to report.

\section{Disclosure}

All authors declare that they have no conflicts of interest.

\section{References}

1. Hasbahceci M, Basak F, Alimoglu O. Cecal volvulus. Indian J Surg. 2012;74(6):476-479. doi:10.1007/s12262-012-0432-9

2. Mahruqi GA, Ebrahim MA, Aghbari SA, et al. Cecal volvulus case report and literature review. Int $J$ Innov Res Med Sci. 2019;4:391-394. doi:10.23958/ijirms/vol04-i06/680

3. Mwita C, Muthoka J, Kanina P, Mulingwa P. Cecal volvulus in an adolescent African male: a case report and brief review of the literature. Pan Afr Med J. 2014;17. doi:10.11604/ pamj.2014.17.92.3946

4. Fenzl N, DeSilva SG, Scott J. Cecal volvulus case report. Imaging Med. 2017;9(2).

5. Rojas CS, Duarte RV, Vivanco DM, Montalvo-Javé EE. Cecal volvulus: a rare cause of intestinal obstruction. Case Rep Gastroenterol. 2020;14:206-211. doi:10.1159/000506933

6. Zabeirou AA, Belghali $\mathrm{H}$, Souiki $\mathrm{T}$, et al. Acute cecal volvulus: a diagnostic and therapeutic challenge in emergency: a case report. Ann Med Surg. 2019;48:69-72. doi:10.1016/j.amsu.2019.10.021

7. Katoh T, Shigemori T, Fukaya R, Suzuki H. Cecal volvulus: report of a case and review of Japanese literature. World $J$ Gastroenterol. 2009;15(20):2547-2549. doi:10.3748/wjg.15.2547

8. Basendowah M, Alabdulqader MH, Abdulqader O, et al. Cecal volvulus post cesarean section: a case report. Cureus. 2020;12(1):e6644. doi: 10.7759 /cureus. 6644

9. Sedik A, Bar EA, Ismail M. Cecal volvulus: a case report and review of literature. Saudi Surg J. 2015;3:47. doi:10.4103/2320-3846.175211

10. Sun G, Walsh B. Cecal volvulus diagnosed with a whirl sign case report. J Educ Teach Emerg MEd. 2020;5(4). doi:10.5070/ M554050328

11. Sharma C, Shekhar S, Kumar S, Chaudhary R. Gangrenous cecal volvulus complicating puerperium: is the delay in diagnosis really inevitable? Case Rep Obstet Gynecol. 2012;2012. doi:10.1155/2012/ 236109

12. Hasbahçeci M, Bafiak F, Kiliç A, et al. Cecal volvulus: report of two cases. Kolon Rektum Hast Derg. 2012;22:21-24.
Open Access Surgery

\section{Publish your work in this journal}

Open Access Surgery is an international, peer-reviewed, open access journal that focuses on all aspects of surgical procedures and interventions. Patient care around the peri-operative period and patient outcomes post-surgery are key topics for the journal. All grades of surgery from minor cosmetic interventions to major surgical procedures are covered. Novel techniques and the utilization of new

Submit your manuscript here: https://www.dovepress.com/open-access-surgery-journa instruments and materials, including implants and prostheses that optimize outcomes constitute major areas of interest. The manuscript management system is completely online and includes a very quick and fair peer-review system, which is all easy to use. Visit http://www.dovepress.com/testimonials.php to read real quotes from published authors. 